This item was submitted to Loughborough's Research Repository by the author.

Items in Figshare are protected by copyright, with all rights reserved, unless otherwise indicated.

\title{
Computer aided modelling of the human spine
}

PLEASE CITE THE PUBLISHED VERSION

http://dx.doi.org/10.1243/0954405991516679

PUBLISHER

Sage / @ IMechE

VERSION

AM (Accepted Manuscript)

LICENCE

CC BY-NC-ND 4.0

REPOSITORY RECORD

Case, Keith, Di-Chen Xiao, B. Serpil Acar, and J. Mark Porter. 2019. "Computer Aided Modelling of the Human Spine". figshare. https://hdl.handle.net/2134/13955. 
This item was submitted to Loughborough's Institutional Repository (https://dspace.lboro.ac.uk/) by the author and is made available under the following Creative Commons Licence conditions.

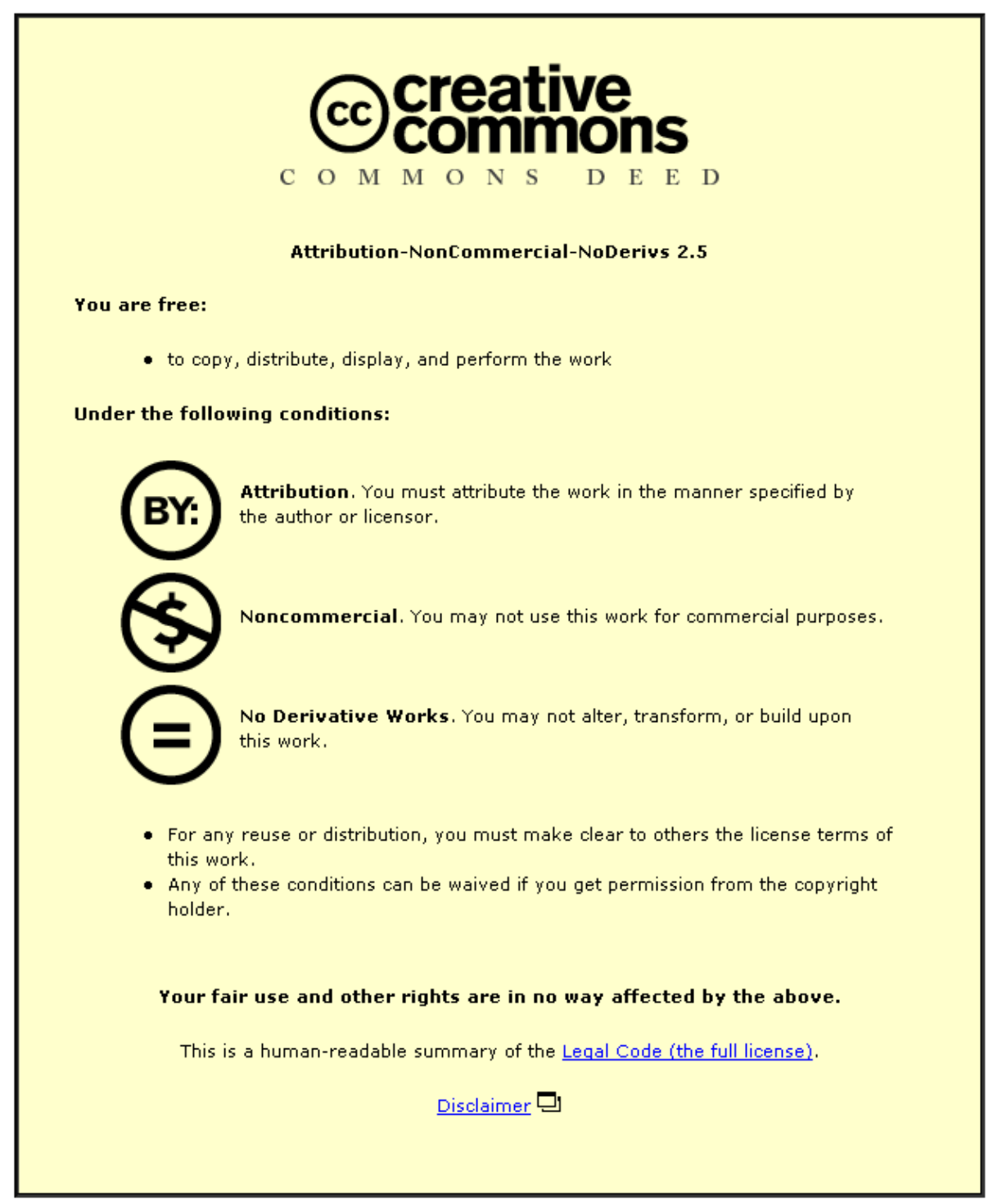

For the full text of this licence, please go to: http://creativecommons.org/licenses/by-nc-nd/2.5/ 


\title{
Computer aided modelling of the human spine
}

\author{
K Case $^{1 *}$, D C Xiao ${ }^{2}$, B S Acar ${ }^{3}$ and J M Porter ${ }^{4}$ \\ ${ }^{1}$ Department of Manufacturing Engineering, Loughborough University, Loughborough, UK \\ ${ }^{2}$ Department of Manufacturing Engineering and Design, University of East London, Dagenham, Essex, UK \\ ${ }^{3}$ Department of Computer Studies, Loughborough University, Loughborough, UK \\ ${ }^{4}$ Department of Design and Technology, Loughborough University, Loughborough, UK
}

\begin{abstract}
The human spine is the main structure to support human body weight and external loads, to allow the torso to reach to a variety of positions and to protect the spinal nervous system. Lumbar back pain and disorders may be related to spinal curvature and disc pressure, and it is an ultimate objective of the work reported here to include consideration of these issues in computer aided ergonomics design systems for evaluating a wide range of situations including manual handling and car seat design.

Several methods from structural analysis have previously been used to model the human spine, principally lever and beam structures, but these have frequently shown discrepancies when compared with experimental data. As an alternative, an arch representation for the spine is considered here and allows the establishment of a criterion for the failure of the spine that may be useful in determining absolute maximum loading conditions. However, the main interest is in submaximal loading conditions where damage or discomfort are the concerns rather than fracture. It is proposed that the location of the thrust line in relation to the centre-line of the spine is a useful predictor, and optimization techniques have been developed to find the 'best-fitting' thrust line for the statically indeterminate structure.

Further work is concerned with adding muscle and ligament forces to the loading system of the model, extension of the two-dimensional model into three dimensions, validation against experimental data and integration with the SAMMIE computer aided ergonomics design system.
\end{abstract}

Keywords: computer aided modelling, human spine, arch, design, loading

\section{INTRODUCTION}

The spine is a three-dimensional mechanical structure that transmits loads, allows motion and protects the spinal cord from damage. The spinal column consists of cervical vertebra, $\mathrm{C} 1$ to $\mathrm{C} 7$, thoracic vertebra, $\mathrm{T} 1$ to T12, lumbar vertebra, L1 to L5, the sacrum and intervertebral discs. The lumbar spine is designed to bear great loads and provide truncal mobility while the discs resist compression and determine mobility. The spinal column system also includes ligaments, attached muscles and the rib cage. The ligaments take tensile loads and the muscles supply forces to maintain spine stability.
An understanding of the spine is important in the study of its normal functional conditions such as osteoporosis, scoliosis and kyphosis, but current understanding is limited by the structural complexity of the spine and the difficulty of in vivo experiments (i.e. with human subjects). Hence many mathematical models of the human spine have been developed, and these are principally classified as levers, simple beams, cantilever beams and arches.

Lever models typically describe the whole spine as a rigid lever without consideration of curvature, and loading of the spine is balanced by reaction forces at the sacrum. Simple beam models describe the whole spine as a straight slender elastic beam, and Euler's theory of elastic buckling is used for analysis. Cantilever models describe the whole spine as a cantilever beam rigidly built into the pelvis with applied forces and moments. Arch models describe either a part or all of the spine as an arch. Stability under a variety of loading conditions can then be determined by 
compliance with the criterion that the thrust line should be completely located within the 'core' of the arch spine.

Intravertebral disc pressure increases with intraabdominal pressure [1], but lever models predict the reverse effect and can produce unrealistically large reaction forces at the sacrum [2]. For example, with these models a $77 \mathrm{~kg}$ man holding a $90.7 \mathrm{~kg}$ weight in a stooped posture leads to a predicted reaction force at the sacrum of $6.6 \mathrm{kN} \mathrm{[3],} \mathrm{which} \mathrm{is} \mathrm{comparable} \mathrm{with} \mathrm{the}$ fracture strength of the end-plates of the vertebral body (whose bearing strength is about $5-8 \mathrm{kN}$ ). Clearly this situation, although well outside normal working practices, is sustainable without damage in the extreme by weightlifters. This discrepancy is considered to arise from the way in which lever models ignore internal forces and spinal curvature. In contrast, modelling the spine as an arch could be a more realistic representation which considers both spinal curvature and internal forces. The increase in disc pressure with increasing intra-abdominal pressure in standing and upright postures [2] can be explained by the introduction of lumbar lordosis and intra-abdominal pressure which acts on the convex surface of the arch lumbar spine. The fact that the disc pressure reduces with increasing intraabdominal pressure in stooping and leaning [1] can be explained by the intra-abdominal pressure acting on the

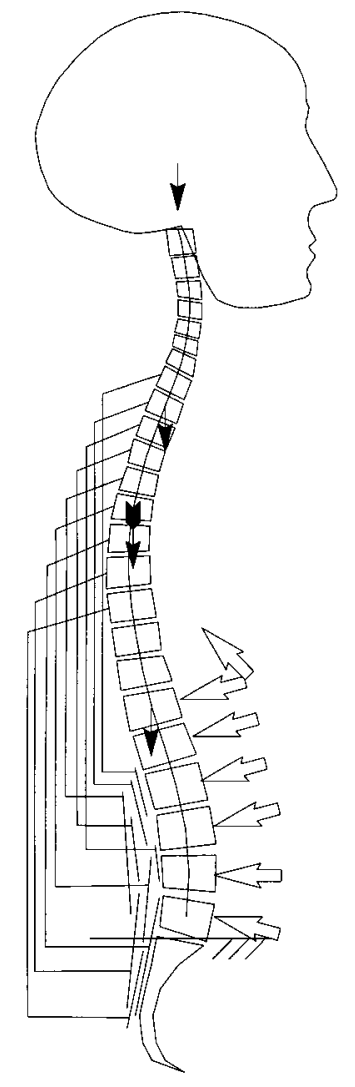

$\downarrow \quad \begin{array}{ll}\text { Body } \\ \text { Weight }\end{array}$

External
Loads

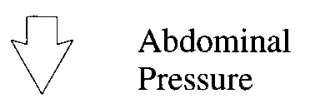

Fig. 1 Loading system of the spine (not all muscle forces shown)
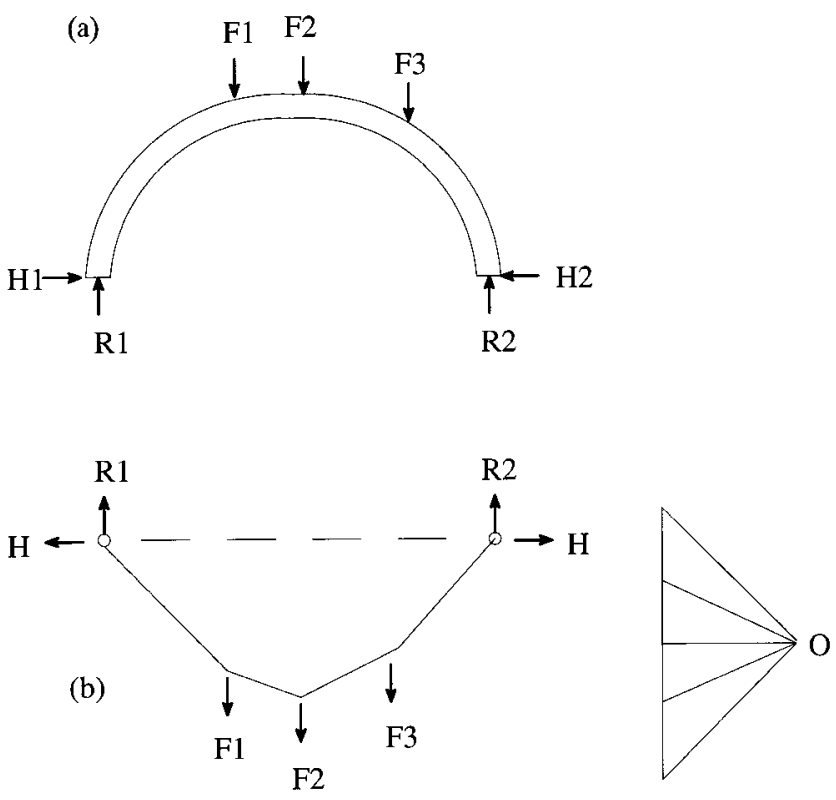

(c)

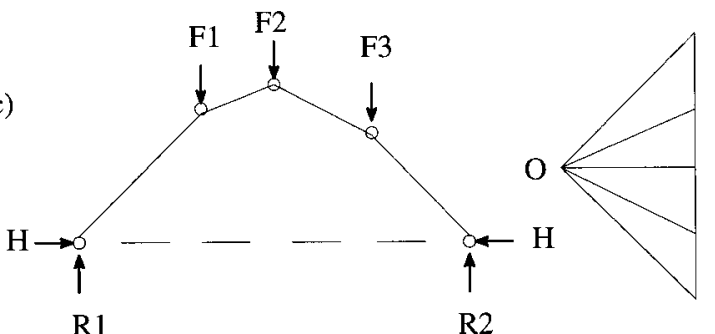

Fig. 2 String and arch in equilibrium (from reference [4]): (a) masonry arch, (b) string in equilibrium, (c) arch in equilibrium

concave surface of the arch spine. These factors can be included in an arch model and result in a reaction force at the sacrum of $1.3-1.5 \mathrm{kN}$ for the loading situation described above.

\section{STATICALLY INDETERMINATE STRUCTURE OF THE SPINE}

The loading system of the human spine can be simplified as body weight, external loads, muscle forces, ligament forces and abdominal pressure, as shown in Fig. 1. The spine structure may be approximated as a tower consisting of independent block layers. Each layer is fixed by a number of strings to the base. This structure is statically indeterminate as the number of unknowns is greater than the number of equations available for their solution. The tension in the strings, the frictional forces between the layers and the connection conditions between the strings and the base all are unknown.

A loaded arch (Fig. 2a) is statically indeterminate, as the reaction forces H1, H2, R1 and R2 cannot be found 
using force and moment equilibrium equations such as L. $f_{x}=0$, L. $f_{y}=0$ and L. $M_{\mathrm{A}}=0$. The funicular polygon is an analysis tool [4] that generates alternative solutions (Fig. 2b). The problems of the hanging string in Fig. 2b and the arch which is modelled as hinged rigid rods in Fig. 2c are the same in statics. The rods work in compression while the strings are in tension. Compressive force, thrust, is transmitted along a line called a thrust line. The thickness of the arch voussoirs which contain a thrust line and the funicular polygon determines the stability of the arch.

If the horizontal component $\mathrm{H}$ ( $\mathrm{H} 1=\mathrm{H} 2$ in this case) is known, then the funicular polygon in Fig. 2c can be drawn from the force polygon. The pole $\mathrm{O}$ could be placed anywhere, so an infinite number of thrust lines corresponding to different pole positions could be found.

Loads applied in general directions are treated in a similar way to vertical loads. The position and shape of the funicular polygon again changes with the pole $\mathrm{O}$ of the force polygon. According to the 'middle-third rule', if the applied load (thrust line) stays within a 'core' of the section, stresses across the whole section will be compressive and the arch is safe. For a rectangular section the core has a depth of one-third of the total depth. The existence of a satisfactory thrust line ensures that the arch cannot collapse, whereas thrust lines outside the arch cause collapse due to the formation of hinges.

The spine can be modelled as an arch with the assumptions that (a) loads are transmitted by compressive forces along the spine, (b) normal compressive forces are lower than the crushing strength of vertebrae and discs and (c) sliding failure cannot occur. The spine is similar to the masonry arch but the factor of safety may be different. Also, the centre-line of the 'core' may not be located at the central line of the vertebrae of the spine, and the diameter of the 'core' may increase due to the actions of ligaments and muscles. A hinge forms as a thrust line approaches the surface of the vertebrae, as shown in Fig. 3a. Posterior extrusion of fibrocartilage from the disc, caused by hydraulic wedging pressure and the stretching of ligaments, may be a cause of back pain, as shown in Fig. 3b (see reference [5]).

\section{FAILURE CRITERION AND THRUST LINE OPTIMIZATION}

An infinite number of thrust lines for an arch exist as it is a statically indeterminate structure. Finding the best (closest to the centre-line of the arch) thrust line is an optimization problem. A criterion of failure for the spine is then:

If : the best-fitting thrust line among all thrust lines in a spine can be found but it is not located within the 'core' of the complete arch spine, Then: the spine fails or disorders occur.

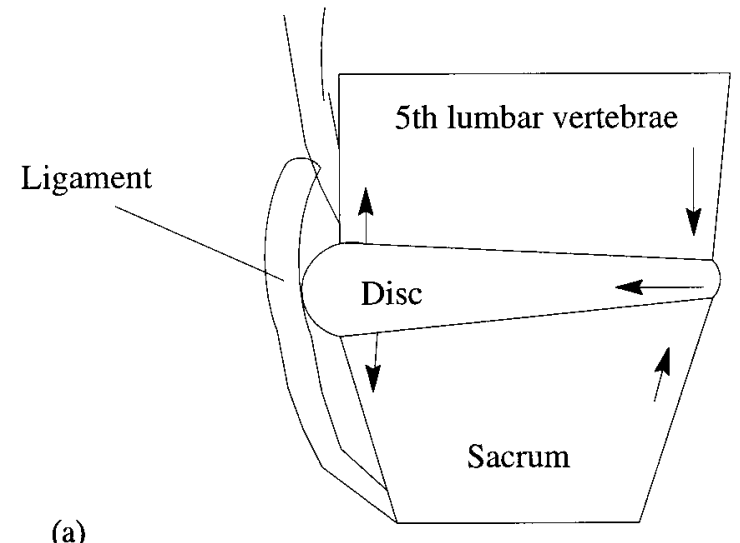

(a)

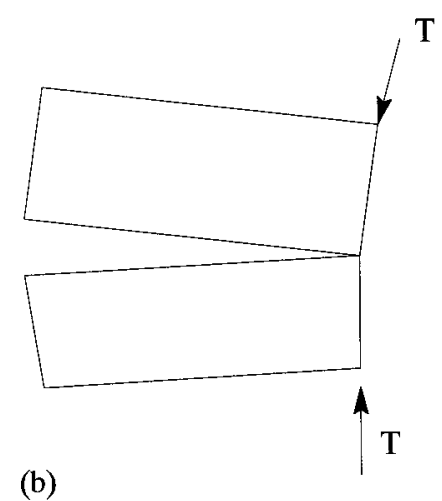

Fig. 3 Failure of the arch spine

To find the best-fitting thrust line it is necessary to find the best position of the pole $\mathrm{O}$ of the force polygon. This is a two-dimensional non-linear optimization problem, and four objective functions to achieve this are presented as follows:

$$
\begin{array}{ll}
f_{1}=\min \left[\max / d_{i} /\right] ; & i=1, n \\
f_{2}=\min \left[\mathrm{L} . d_{i}^{2}\right] ; & i=1, n \\
f_{3}=\min \left[\mathrm{L} .\left(w_{i} / d_{i} /\right)\right] ; & i=1, n \\
f_{4}=\min \left[w_{1} / d_{n} /+w_{2} \max / d_{i} /\right] ; & i=1, n-1
\end{array}
$$

where $/ d /$ is the distance between the thrust line and the spine central line, $w$ is a weighting factor which forces the thrust line to be as close as possible to the central line and $n$ is the number of forces. The best result of several locally optimized positions of the pole $\mathrm{O}$ in the force polygon from the above four objective functions is chosen as the final optimization result.

\section{CASE STUDY}

The forward bending, heavy load problem in reference [6] was taken as a calculation example. A person in a stooped posture carrying a $900 \mathrm{~N}$ load in both hands and 


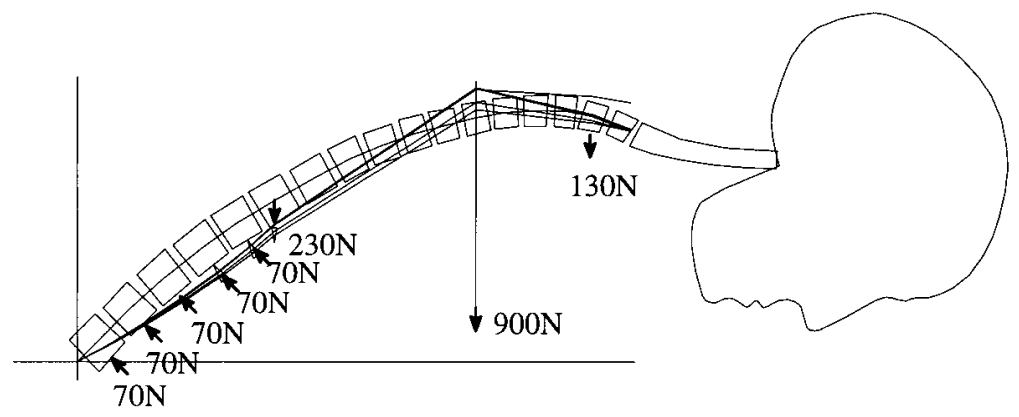

Fig. 4 Optimization of the thrust line

which acts on T6, a head and shoulder weight of $130 \mathrm{~N}$ acting on T2, a trunk weight of $230 \mathrm{~N}$ acting on T12, intra-abdominal pressure of $70 \mathrm{~N}$ acting perpendicularly to each of the vertebrae L1 to L5 were modelled as shown in Fig. 4. The body weight, intra-abdominal pressure and additional load carried are treated as the external loads, and the ligament and muscle forces are treated as internal forces. The optimization calculation results are shown in Fig. 4. Comparison between the optimization result and the result from reference [1] reveals that the optimized thrust line is closer to the spine centre-line.

\section{CONCLUSIONS}

The reaction force at the sacrum calculated by the elastic lever model was about $6.6 \mathrm{kN}$, which is comparable with that capable of rupturing the end-plates and causing crushing of the vertebral bone $(5-8 \mathrm{kN})$. Compared with the lever model, the predicted reaction force calculated by the arch model with optimization is approximately $1.3-1.6 \mathrm{kN}$, which is a more realistic evaluation of the situation.

A criterion of failure of the spine is generated in a situation where, if the best-fitting thrust line among all of the thrust lines in a spine can be found but it is not located within the 'core' of the complete spine, then the spine fails or disorders occur. The best-fitting thrust line which is as close to the reference line of the spine as possible can be obtained using optimization techniques and is a better predictor compared with those reported in the literature.

\section{ACKNOWLEDGEMENTS}

The research was funded by the Engineering and Physical Sciences Research Council under Grant GR/ K58241, 'Spine Modelling: A Tool for Safe to Use Equipment Design'.

\section{REFERENCES}

1 Nachemson, A. L. Valsalva manoeuvre biomechanics effects on lumbar trunk loads of elevated intra-abdominal pressures. Spine, 1986, 11(5), 476-479.

2 Morris, J. M., Lucas, D. B. and Bresler, B. Role of the trunk in stability of the spine. J. Bone Jt Surg., April 1961, 43-A (Am. Vol.), 327-351.

3 Macintosh, J. E. and Bogduk, N. The attachments of the lumbar erector spinae. Spine, 1991, 16(7), 783-792.

4 Heyman, J. The Masonry Arch, 1982 (Ellis Horwood, New York).

5 Keegan, J. J. Alterations of the lumbar curve related to posture and seating. J. Bone Jt Surg., July 1953, 35-A(3), 589-603.

6 Aspden, R. M. The spine as an arch: a new mathematical model. Spine, 1989, 14, 266-274. 